\title{
THE VARIATION IN WAKE STRUCTURE OF A TIDAL STREAM TURBINE WITH FLOW VELOCITY
}

\author{
MARINE 2011 \\ R. MALKI, I. MASTERS, A.J. WILLIAMS AND T.N. CROFT \\ Marine Energy Research Group, Civil and Computational Research Centre, Swansea University \\ Swansea, United Kingdom \\ e-mail: r.malki@swansea.ac.uk, web page: http://www.swan.ac.uk/engineering/marineenergy/
}

Key words: BEM, CFD, tidal stream turbines, wakes.

Summary. A combined Blade Element Momentum - Computational Fluid Dynamics (BEM-CFD) model is applied to a $10 \mathrm{~m}$ diameter tidal stream turbine blade and the supporting nacelle and tower structure in a $700 \mathrm{~m}$ long rectangular channel. The modelling approach is computationally efficient and is suitable for capturing the time-averaged influence of the turbine on the flow. A range of simulations are conducted for the purpose of undertaking a comparative study of the influence of the turbine on mean flow characteristics. Variations in flow structure around the turbine for different flow conditions were evaluated.

Simulations are conducted for a range free-stream velocities typical of potential tidal stream deployment sites, typically up to $3.0 \mathrm{~m} \mathrm{~s}^{-1}$. Velocity deficit profiles and wake dimensions are evaluated for each flow condition implemented. Downstream flow recovery is strongly linked to the flow velocity, and occurs over a longer distance with increasing velocity. For the range of velocities considered, some properties, such as wake length and the maximum wake length location increase linearly, or nearly-linearly with velocity. Other properties, such as the maximum wake width, and the recovery distance downstream demonstrate a tendency to converge towards a constant value. The key findings of this study highlight the significance of the free-stream velocity as an influence on the flow structure around and downstream of a tidal stream turbine.

\section{INTRODUCTION}

The extraction of tidal stream energy is a relatively new concept although it draws upon the wide knowledge base developed for the wind power industry. Nevertheless, there is much uncertainty associated with tidal power due to the significant contrasts between atmospheric and aquatic environments, for example the differences in fluid density and viscosity. These properties have implications on the loads experienced by the devices as well as flow Reynolds numbers.

A number of deployment initiatives are currently underway to investigate the performance of tidal stream turbines in offshore environments ${ }^{1-3}$. Although the outcomes from such initiatives will be very valuable, these deployments are very costly and there can be significant risks to project development investments due to the highly dynamic flows which 
are likely to occur at the most suitable sites for exploiting tidal stream energy. Understanding how turbine designs are likely to perform in various conditions can be useful at the planning stage to maximise the return on the investment without subjecting the devices to avoidable damage. This is particularly important at the early test trial stages on which the fate of future and more significant scaled-up deployments for large scale power generation may depend.

Future deployments are likely to consist of numerous tidal stream turbines being deployed in predetermined configurations. To maximise the power generation potential per device, blade diameters will be significant to increase their projected area of influence across the flow, and hence, turbine wakes will be large. Ideally, the spacing between the devices should be minimised to maximise the number of devices deployed across a given site without compromising the performance of the devices. This could occur if the devices are too close to each other and hence, turbine blades fall within the wakes generated by upstream or neighbouring turbines. Ultimately, the economics will be the deciding factor when determining the number of devices to deploy. A loss in efficiency is acceptable if more devices increase profits. However, an understanding of wake structures around the turbines is necessary to aid the planning process.

To numerically simulate turbine blades, a moving mesh approach would be suitable for capturing the transient nature of the flow as it interacts with the blades ${ }^{4}$. However, this approach is very computationally expensive. An alternative method, which is presented and used here, is a combined blade element momentum - computational fluid dynamics (BEMCFD) model where the time-averaged forces acting on the flow due to the blades are introduced into the flow domain in the form of momentum sink terms.

The majority of tidal stream turbine studies to date have focused on the performance of the devices and power outputs in hypothetical flow conditions ${ }^{5}$. An investigation into the wake structure downstream of a turbine and how it varies with flow velocity is presented in this paper to assist the planning of tidal stream turbine array layouts.

\section{The BEM-CFD Model}

\subsection{Governing Equations}

The steady-state CFD model solves the Navier-Stokes Equations, which consist of the equations for mass continuity (1) and the conservation of momentum (2) whereby the fluid is treated as incompressible and turbulent.

$$
\begin{gathered}
\nabla \cdot u=0 \\
\nabla \cdot\left(\rho u u_{i}\right)=-\frac{\partial p}{\partial x_{i}}+\nabla \cdot\left(\mu_{\text {lam }}+\mu_{t}\right) \nabla u_{i}+S_{i}
\end{gathered}
$$

In these equations, $\rho$ is the fluid density, $u_{i}$ is the i'th component of the velocity vector, $p$ is the pressure, $\mu_{\text {lam }}$ and $\mu_{t}$ are the dynamic laminar and turbulent viscosities respectively and 
$S_{i}$ represents additional source terms into the momentum equations. Turbulence in the flow is resolved using the $k-\varepsilon \operatorname{model}^{6}$ in which (3) and (4) are solved:

$$
\begin{gathered}
\nabla .(\rho u k)=\nabla \cdot\left(\mu_{\text {lam }}+\frac{\mu_{t}}{\sigma_{k}}\right) \nabla k+\mu_{t} G-\rho \varepsilon \\
\nabla .(\rho u \varepsilon)=\nabla \cdot\left(\mu_{\text {lam }}+\frac{\mu_{t}}{\sigma_{\varepsilon}}\right) \nabla \varepsilon+\frac{\varepsilon}{k}\left(C_{1 \varepsilon} \mu_{t} G-C_{2 \varepsilon} \rho \varepsilon\right)
\end{gathered}
$$

(3) is the conservation equation for turbulent kinetic energy, $k$, and (4) is the conservation equation for the turbulent dissipation rate, $\varepsilon$. The turbulent viscosity, $\mu_{t}$, is calculated using (3) and (4) as follows:

$$
\mu_{t}=\frac{\rho C_{\mu} k^{2}}{\varepsilon}
$$

In (3), (4) and (5), $\sigma_{k}, \sigma_{\varepsilon}, C_{1 \varepsilon}, C_{2 \varepsilon}$ and $C_{\mu}$ are constants for which standard values are used, and $G$ is the turbulent generation rate.

\subsection{Blade Element Momentum Method}

For long time scales, the time-averaged influence of turbine blades on the flow is considered to act over all parts of a circular area with a diameter equal to that of the blade. Forces acting on the flow due to the blade are assumed to be equal across the circular area for equal radial distances from the centre. This time-averaged approach allows sources that represent the force on the fluid due to the blades of the turbines to be applied to each of the momentum equations. The advantage of this approach is that the physical characteristics of the blade are built into the source rather than the mesh consequently allowing the use of better quality meshes. The disadvantage is that because of the time average principle of the approach it fails to resolve any transient flow features due to blade position.

To define the characteristics of the rotor according to axial and radial position the blade element momentum (BEM) $)^{5}$ method is employed. Figure 1a shows diagrammatically how a three bladed rotor is discretised using the BEM approach. The blade properties for a blade element at a certain radius, $r$, are determined and are then averaged throughout the whole of the shaded region. This is performed for each blade element throughout the radius of the rotor, $R$.

Each blade element has a chord length, $c_{F b}$, and radial width, $\delta r$. Each element experiences forces acting on it due to the fluid. These are shown in Figure $1 \mathrm{~b} . \mathrm{d} T$ and $\mathrm{d} F_{A}$ represent the torque and axial forces respectively. The lift, $\mathrm{d} L$, and $\mathrm{drag}, \mathrm{d} D$, are dependent on the angle of attack, $\alpha$, between the blade element and the resultant velocity, $v_{R} . \Omega$ is the angular velocity and $U$ is the upstream longitudinal flow velocity. The chord inclination angle and flow inclination angle are denoted by $\beta$ and $\phi$ respectively. 

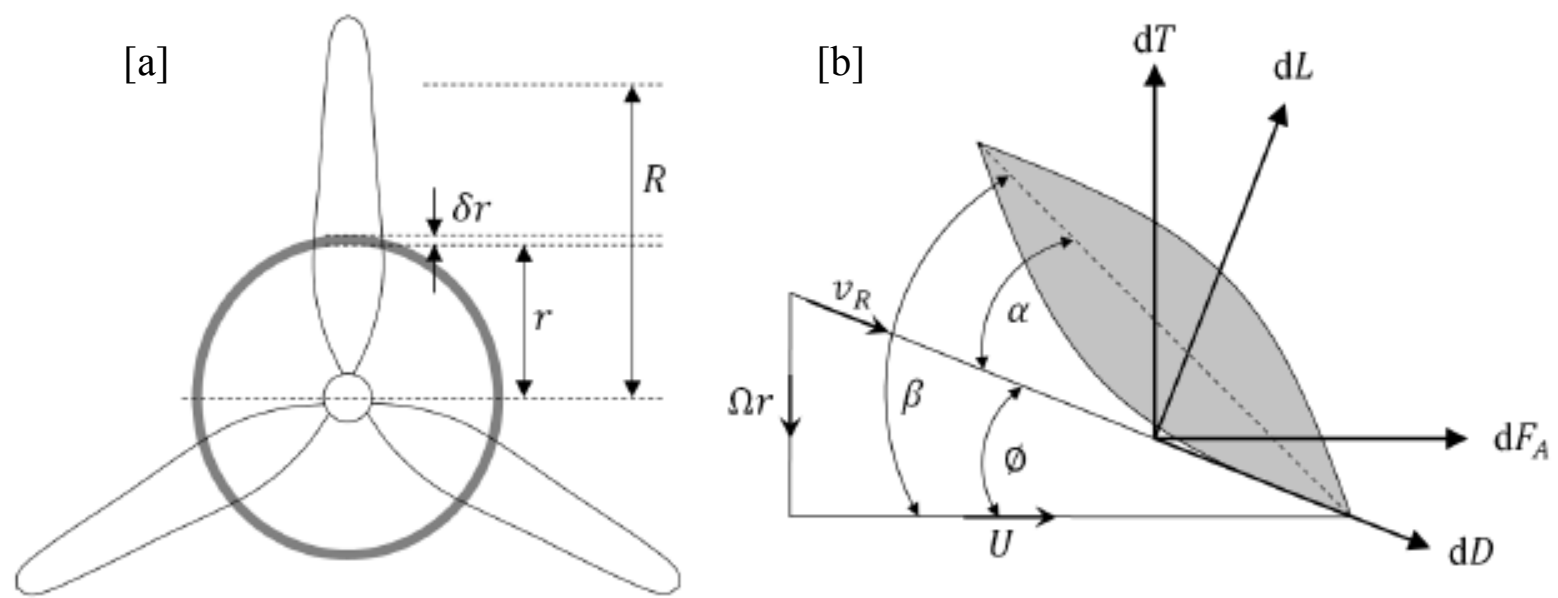

Figure 1: a: Schematic diagram of the discretisation of the rotor; b) resolution of the lift and drag forces

Following the approach described by Masters et al. ${ }^{5}$, an axial force on a blade element is defined by:

$$
\mathrm{d} F_{A}=\mathrm{d} L \sin \phi+\mathrm{d} D \cos \phi
$$

and the tangential force on a blade element, which is equal to the torque/radius, i.e. $\mathrm{d} T / r$, is defined by:

$$
\mathrm{d} F_{T}=\mathrm{d} L \cos \phi-\mathrm{d} D \sin \phi
$$

The flow inclination angle, $\phi$, is defined by:

$$
\phi=\tan ^{-1}\left(\frac{\Omega r-u_{\theta}}{u_{z}}\right)
$$

where $u_{\theta}, u_{z}$ are the local fluid tangential and axial velocities respectively, and $\Omega$ is the angular velocity of the blades in $\mathrm{rad} / \mathrm{s}$. The lift force, $\mathrm{d} L$, and drag force, $\mathrm{d} D$, are given by:

$$
\begin{aligned}
& \mathrm{d} L=\frac{1}{2} \rho\left|v_{R}\right|^{2} C_{L} c_{F b} \delta r \\
& \mathrm{~d} D=\frac{1}{2} \rho\left|v_{R}\right|^{2} C_{D} c_{F b} \delta r
\end{aligned}
$$

Here, $C_{L}$ and $C_{D}$ are the lift and drag coefficients respectively, and:

$$
\left|v_{R}\right|^{2}=u_{z}^{2}+\left(\Omega r-u_{\theta}\right)^{2}
$$

Substituting (9) and (10) into (6) and (7) gives the following equations:

$$
S_{z}=\mathrm{d} F_{A}=\frac{1}{2} \rho\left|v_{R}\right|^{2} c_{F b} \delta r\left(C_{L} \sin \phi+C_{D} \cos \phi\right)
$$




$$
S_{\theta}=\mathrm{d} F_{T}=\frac{1}{2} \rho\left|v_{R}\right|^{2} c_{F b} \delta r\left(C_{L} \cos \phi-C_{D} \sin \phi\right)
$$

which, when resolved into Cartesian components and converted into force per volume, are substituted into the momentum equations (2) through $S_{i}$.

\subsection{Computational Solution}

The solution to the governing equations (1)-(4) is achieved using a collocated cell-centred finite volume scheme ${ }^{7}$ within the software PHYSICA $^{8}$. In the finite volume scheme, the physical domain is represented by a mesh consisting of non-overlapping polyhedral elements. In the cell-centred scheme, a solution node is placed at the centre of each of these elements. The governing equations are integrated over each mesh element, and the discretised equations lead to a series of relationships between the unknown values in an element and the values in neighbouring elements. The solution procedure is based on a variant of the SIMPLE $^{9}$ algorithm, and since a collocated scheme is used, the Rhie-Chow approximation is used to prevent checker-boarding of the pressure field ${ }^{10}$. The CFD equations are typically solved for using a diagonally preconditioned conjugate gradient algorithm. Further details of the solution procedure can be found in ${ }^{8,11}$.

The model domain used for the simulations was $700 \mathrm{~m}$ long, $100 \mathrm{~m}$ wide and $30 \mathrm{~m}$ deep. A $10 \mathrm{~m}$ diameter blade was centred laterally and vertically across the channel, and positioned $300 \mathrm{~m}$ from the upstream boundary. This is to ensure the full development of the boundary layer and the achievement of a uniform flow upstream of the turbine. Also, a considerable distance is required downstream of the turbine to evaluate the development of the flow.

An element size of $0.19 \mathrm{~m}$ by $0.19 \mathrm{~m}$ by $0.1 \mathrm{~m}$ was implemented within the blade box. This is the region within which the momentum sink terms associated with the blade are introduced, and hence, a relatively fine mesh is required to capture the variation in lift and drag forces across the blade. The element sizes were gradually increased away from the blade box to reduce the overall mesh size, however, to a lesser extent within the wake region downstream of the blade where the flow structure is to be studied.

Each simulation was carried out for 15,000 iterations, which required 44 hours of computation on a single processor. However, the simulations were conducted in parallel on a multi-node cluster and hence, actual wall-times were significantly reduced.

\subsection{Definitions}

The tip speed ratio (TSR), the ratio between the rotational speed of the tip of the blades and the free-stream longitudinal flow velocity, is defined by:

$$
\mathrm{TSR}=\frac{D \Omega}{2 U}
$$

where $U$ is the upstream free velocity and $D$ is the turbine diameter. 
The 'velocity deficit', $U_{d e f}$, is used in this paper in accordance with the definition suggested by Myers and Bahaj ${ }^{12}$ as follows:

$$
U_{d e f}=1-\frac{u}{U}
$$

such that a velocity deficit of 0.25 for example, is equivalent to a local velocity equivalent to $75 \%$ of the free-stream velocity.

\section{Results and Discussion}

\subsection{Simulations}

The simulations presented in this paper were conducted for a tip speed ratio of 3.0, which is approximately the optimum for the blade used. A range of velocities typical of offshore environments where tidal stream turbines are likely to be deployed were implemented, typically ranging up to $3.0 \mathrm{~m} \mathrm{~s}^{-1}$. Since power is a function of the cubic power of velocity, there is a significant amplification of power output with increasing velocity (see Figure $2 b$ ).

To fully appreciate the significance of this curve, consider a typical diurnal tidal cycle consisting of two periods of high tide annexed together by an intermediate period of low tide. Maximum velocities may be expected during a 'turning' tide which occurs between low and high tides. However, during a slack tide, which occurs around low and high tides, there is relatively little flow movement. When conducting feasibility studies, tidal energy developers may be tempted to estimate potential power outputs using a rated velocity factor of $70 \%{ }^{13}$, based on the maximum flow velocities observed during a mean spring tide across a potential deployment site. However, to gain a more accurate appreciation of the true return of the investment, a more detailed assessment of the power outputs over the range of velocities, and range of tidal cycles would be required. Velocities will not only vary across each tidal cycle, but also between different tidal cycles due to a natural variation in low and high tide sizes, in addition to the influence of meteorological factors, particularly wind-generated waves.

\subsection{Downstream Velocity Deficit}

The reduction in velocity deficit with downstream distance from a turbine along the flow axis through the centre of the blade is a measure of flow recovery following the momentum extraction and velocity reduction arising due to the interaction of the blade with the flow. This can be used to determine suitable longitudinal distances between turbines within an array to minimise wake interference between the devices, and hence, avoid compromising the performance of downstream devices.

Velocity deficit profiles downstream of the blade along its centreline are presented in Figure $3 \mathrm{a}$ for the range of inlet velocities considered. Initially, the velocity deficit profiles are similar in magnitude, although they are slightly greater in magnitude for the lowest velocities, particularly the lowest velocity of $0.5 \mathrm{~m} \mathrm{~s}^{-1}$. The profiles level off with downstream distance towards a constant value of 0.01 . The velocity actually recovers to the free-stream velocity 
(i.e. a velocity deficit of zero), however, the free-stream velocity is estimated here based on the velocity two diameters upstream of the blade, hence the slight surplus in the final velocity deficit. This occurs at different rates, and the minimum value is reached over a longer downstream distance with increasing velocity. The profiles indicate some minor meshdependency issues, particularly in far regions downstream, where the mesh is relatively coarser than near the turbine.
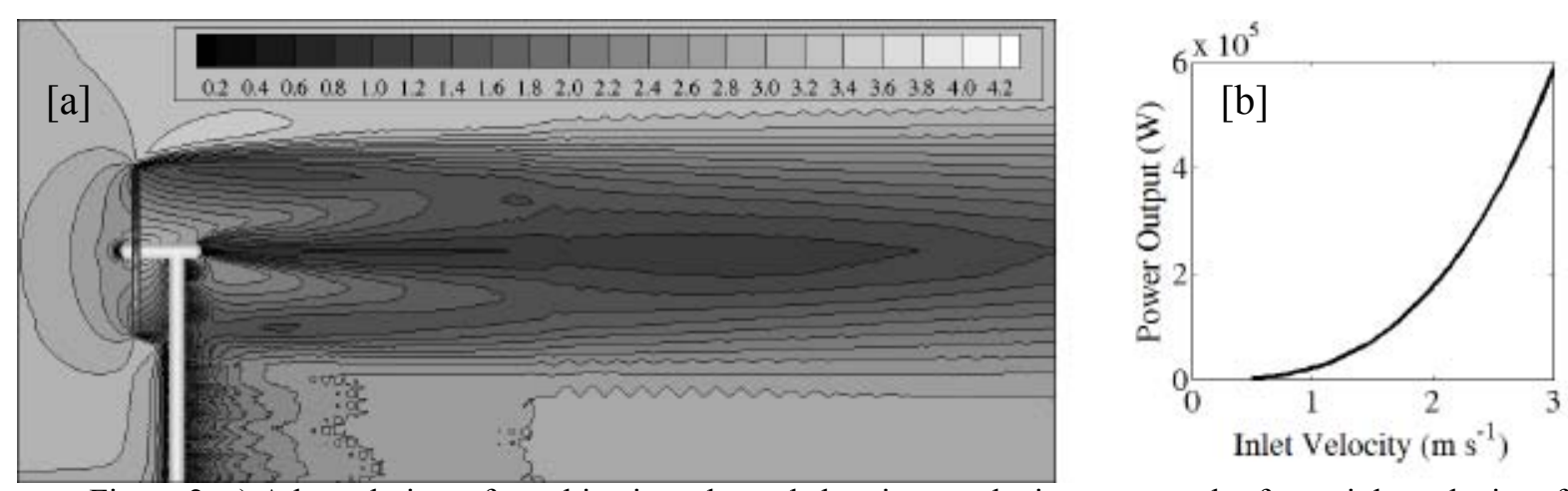

Figure 2: a) A lateral view of a turbine in a channel showing a velocity contour plot for an inlet velocity of $3.0 \mathrm{~m} \mathrm{~s}^{-1}$; and b) variation in power output with inlet velocity. Tip speed ratio is 3.0

To evaluate flow recovery downstream of the turbine, the distances required for the velocity along centreline through the middle of the blade to return to velocity deficits of 0.05 , 0.15 and 0.25 are considered in Figure $3 \mathrm{~b}$. The relationship between the distance required for a recovery to 0.1 of the free-stream velocity and the inlet velocity is linear and the recovery distance continues to increase at the maximum velocity considered of $3.0 \mathrm{~m} \mathrm{~s}^{-1}$. Recovery to lower velocity deficits, for example 0.15 or 0.25 , departs from a linear trend to a point where the recovery distance does not appear to increase with increasing velocity. This can be seen most clearly for the recovery to a velocity deficit of 0.25 whereby the recovery distance shows little variation for velocities above $2.5 \mathrm{~m} \mathrm{~s}^{-1}$, and the recovery distance is approximately 16.4 diameters.

\subsection{Lateral Velocity Deficit Profiles}

The influence of a turbine on the flow structure is not restricted to the flow axis through the centre of the turbine, but also affects the adjacent regions of the flow. An understanding of this scope of influence is required to aid in the planning and placement of multiple turbines within an array. To assess this aspect, velocity deficit profiles are considered for lateral sections perpendicular to the flow direction.

Lateral profiles are presented at a range of downstream distances from the turbine blade for flow velocities of $1.0 \mathrm{~m} \mathrm{~s}^{-1}$ and $3.0 \mathrm{~m} \mathrm{~s}^{-1}$ in Figure $4 \mathrm{a}$ and $\mathrm{b}$ respectively. These represent velocities around the lower and upper end of the operating range of velocities in a typical offshore environment suitable for tidal stream turbine deployment. In both flows, there is initially little difference in the velocity deficit profiles with downstream distance up to 4.0 diameters downstream from the blade. Within this range, the velocity deficit is significant. 

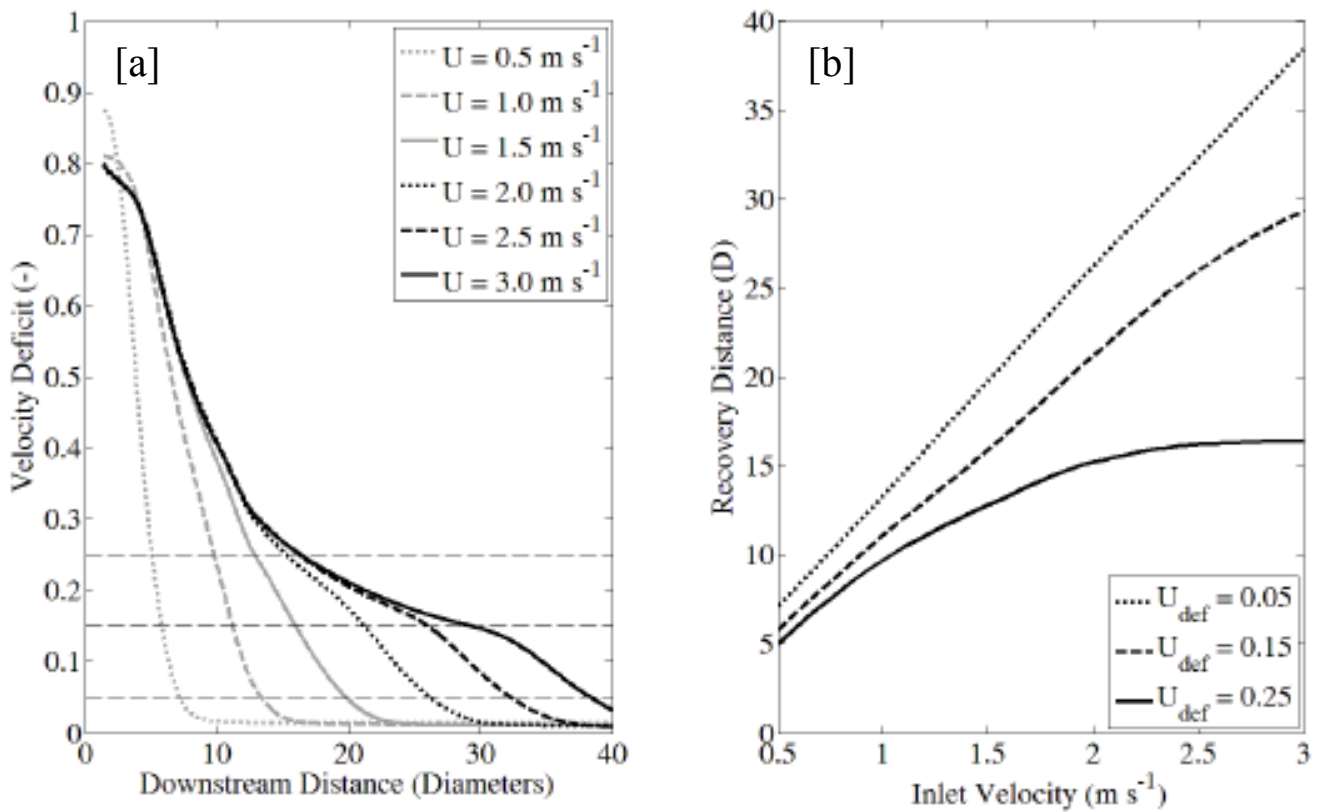

Figure 3: a) Velocity deficit profiles downstream of a $10 \mathrm{~m}$ diameter turbine for inlet velocities between 0.5 and $3.0 \mathrm{~m} \mathrm{~s}^{-1}$. b) Downstream distance required for recovery to a velocity deficit of $0.05,0.15$ and 0.25 for the range of velocities considered. Tip speed ratio is 3.0
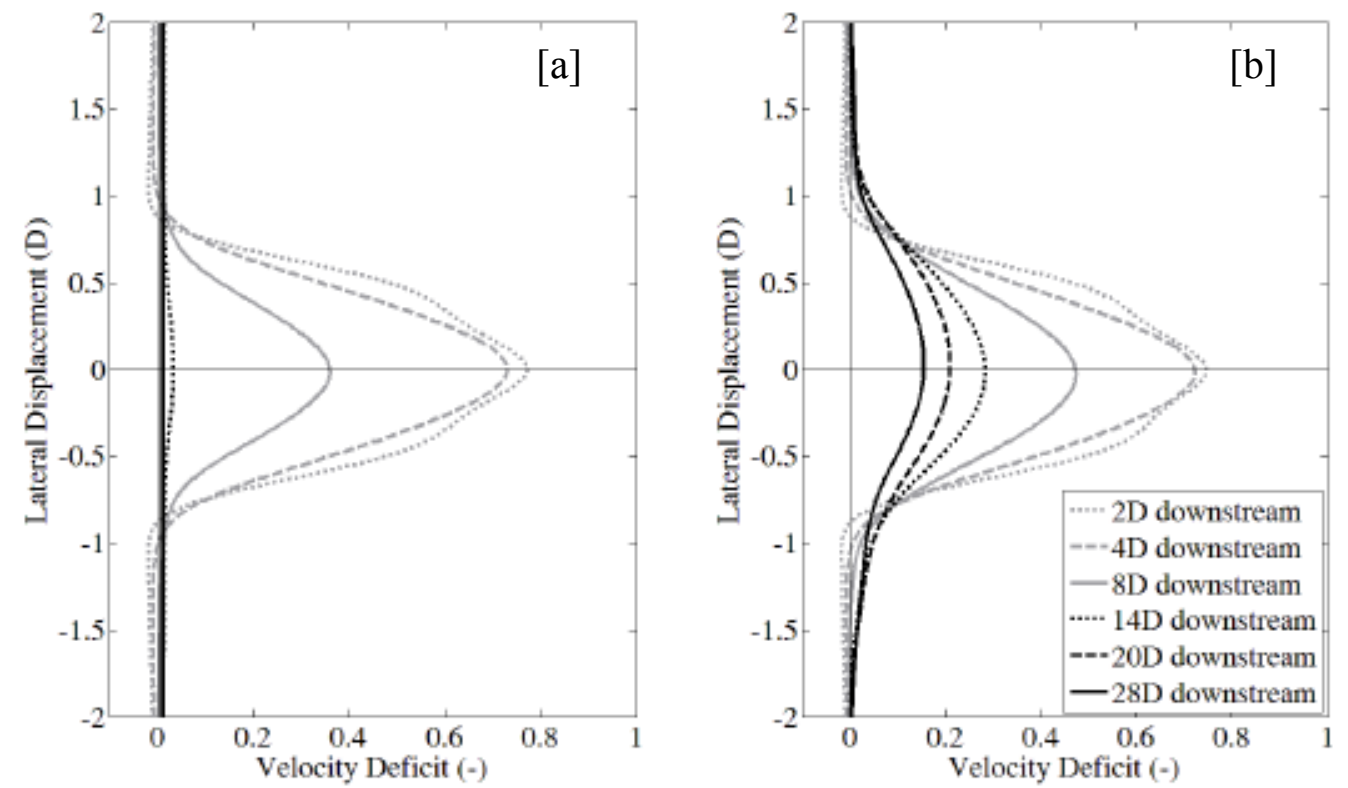

Figure 4: Lateral profiles of velocity deficit downstream from a $10.0 \mathrm{~m}$ diameter turbine for inlet velocities of a) $1.0 \mathrm{~m} \mathrm{~s}^{-1}$; and b) $3.0 \mathrm{~m} \mathrm{~s}^{-1}$. Tip speed ratio is 3.0 .

Beyond 4.0 diameters downstream, there is a sharp decrease in velocity deficit, particularly for a lower flow velocity, where the velocity deficit is negligible beyond 8.0 diameters 
downstream. For the higher flow velocity, there is a steady decrease in velocity deficit with distance downstream beyond 4.0 diameters, although the influence of the turbine is still evident beyond 28.0 diameters downstream.

Now lateral profiles of velocity deficit are compared for different inflow velocities at two different sections: 2.0 and 10.0 diameters downstream from the blade (Figure 5a and b respectively). Immediately downstream of the blade, there is little difference in velocity deficit profiles for different inflow velocities. Velocity deficits are slightly higher for the lowest velocities considered, particularly $0.5 \mathrm{~m} \mathrm{~s}^{-1}$, and to a lesser extent, $1.0 \mathrm{~m} \mathrm{~s}^{-1}$.

At 10.0 diameters downstream, there is little difference in the lateral velocity deficit profiles for inlet velocities above $1.5 \mathrm{~m} \mathrm{~s}^{-1}$. A slightly lower velocity deficit is observed with a velocity of $1.5 \mathrm{~m} \mathrm{~s}^{-1}$, and this decreases significantly for a velocity of $1.0 \mathrm{~m} \mathrm{~s}^{-1}$. For an inlet velocity of $0.5 \mathrm{~m} \mathrm{~s}^{-1}$, the velocity deficit is negligible.
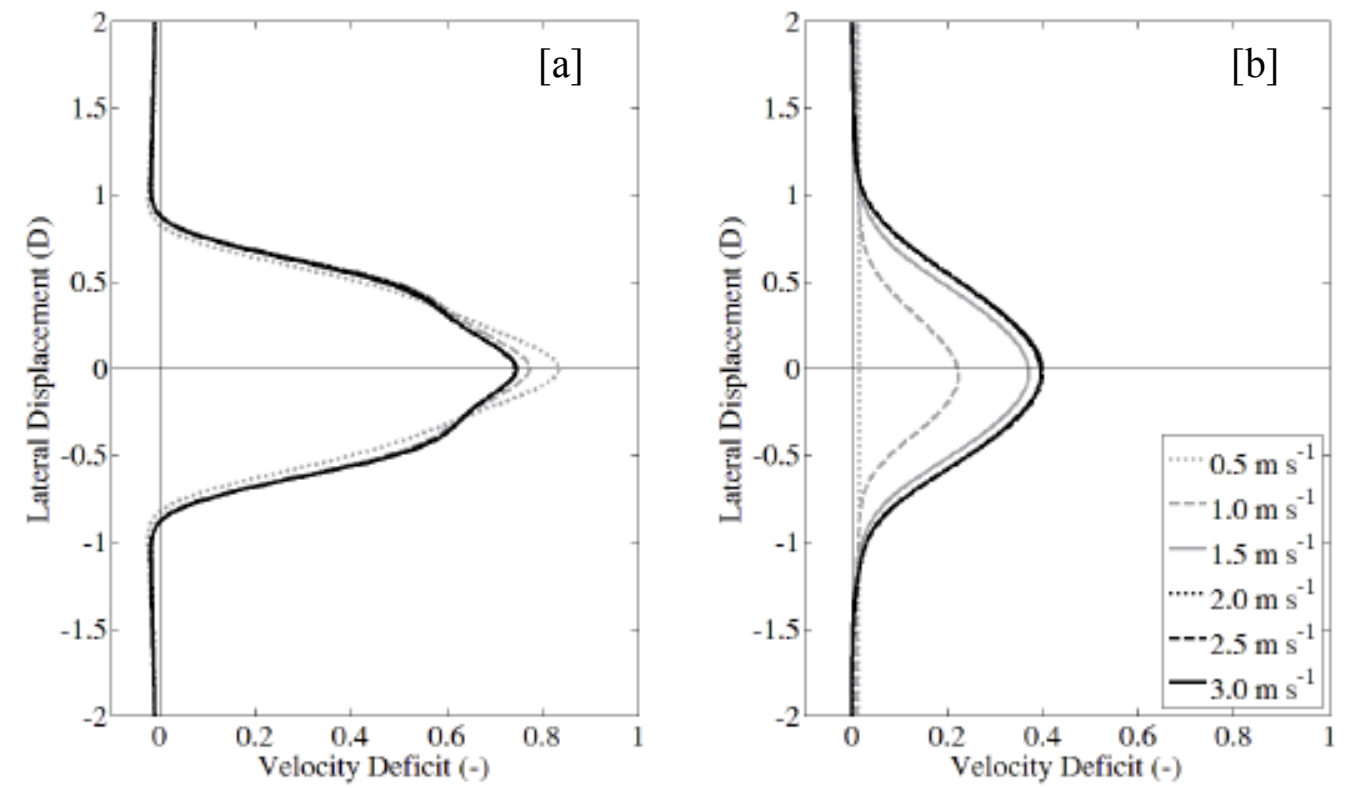

Figure 5: Lateral profiles of velocity deficit at a downstream distance of a) 2.0 diameters; and b) 10 diameters from a $10.0 \mathrm{~m}$ diameter turbine for inlet velocities between 0.5 and $3.0 \mathrm{~m} \mathrm{~s}^{-1}$. Tip speed ratio is 3.0 .

\subsection{Wake Diameter Profiles}

The turbulent wake region arising downstream of a tidal stream turbine may have environmental implications as well as an influence on the performance of neighbouring devices. It is therefore useful to quantify the total area of influence of the wake. The wake edge is defined as the contour around and downstream of the turbine where the velocity is $95 \%$ of the inlet velocity. The wake outlines are presented in Figure 6a for the range of inlet velocities considered. The lack of symmetry observed for higher inlet velocities can be linked to the wakes being longer and hence, extending into a region where the mesh is coarse.

To evaluate wake sizes, diameters were determined by calculating the lateral distance between the two outer edges of the wake along the horizontal plane at hub-level, and this was 
performed at $1.0 \mathrm{~m}$ increments over the length of the wake. Wake diameter profiles are presented in Figure $6 \mathrm{~b}$ for the range of inlet velocities considered. The wake size, both in terms of total length and maximum diameter, are linked to the Reynolds number of the flow, as dictated by the inlet velocity. Wake expansion is initially evident downstream of the turbine in all cases, followed by a rapid reduction due to wake dissipation. Wake expansion continues over a considerably greater distance downstream of the blade with an increasing free-stream velocity.
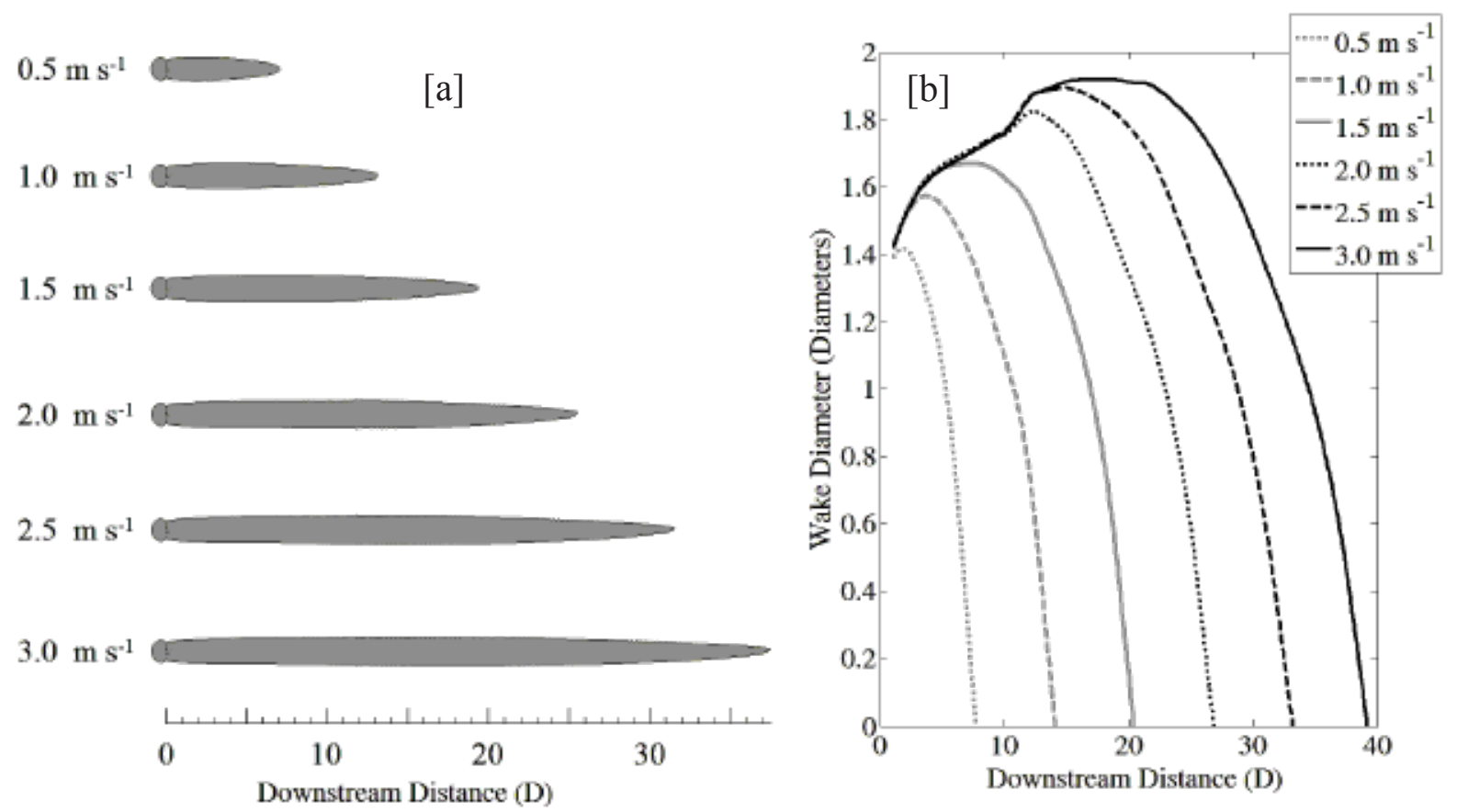

Figure 6: a) Wake outlines based on a wake edge velocity of $95 \%$ of the inlet velocity for inlet velocities between 0.5 and $3.0 \mathrm{~m} \mathrm{~s}^{-1}$; and $\mathrm{b}$ ) wake diameter profiles downstream of a $10 \mathrm{~m}$ diameter turbine for inlet velocities between 0.5 and $3.0 \mathrm{~m} \mathrm{~s}^{-1}$. Tip speed ratio is 3.0

The increases in wake length and diameter with inlet velocity are summarised in Figure 7a and $b$ respectively. A significant increase in wake length occurs. The relationship is linear and equates to approximately $80.0 \%$ of the increase in velocity. Increases in the maximum wake diameter were less significant ranging between 1.42 and 1.92 diameters for velocities between $0.5 \mathrm{~m} \mathrm{~s}^{-1}$ and $3.0 \mathrm{~m} \mathrm{~s}^{-1}$. There was less of an increase in wake diameter with velocity at the higher velocities considered indicating that there is a limit to the increase in the diameter of the wake.

Wake expansion is observed in the simulation results. The expansion of the wake continues over a longer downstream distance with increasing velocity as shown in Figure 7c. Despite the maximum wake width showing less variation with velocity at higher inlet velocities as shown in Figure $7 b$, its location continues to move further downstream from the blade. 

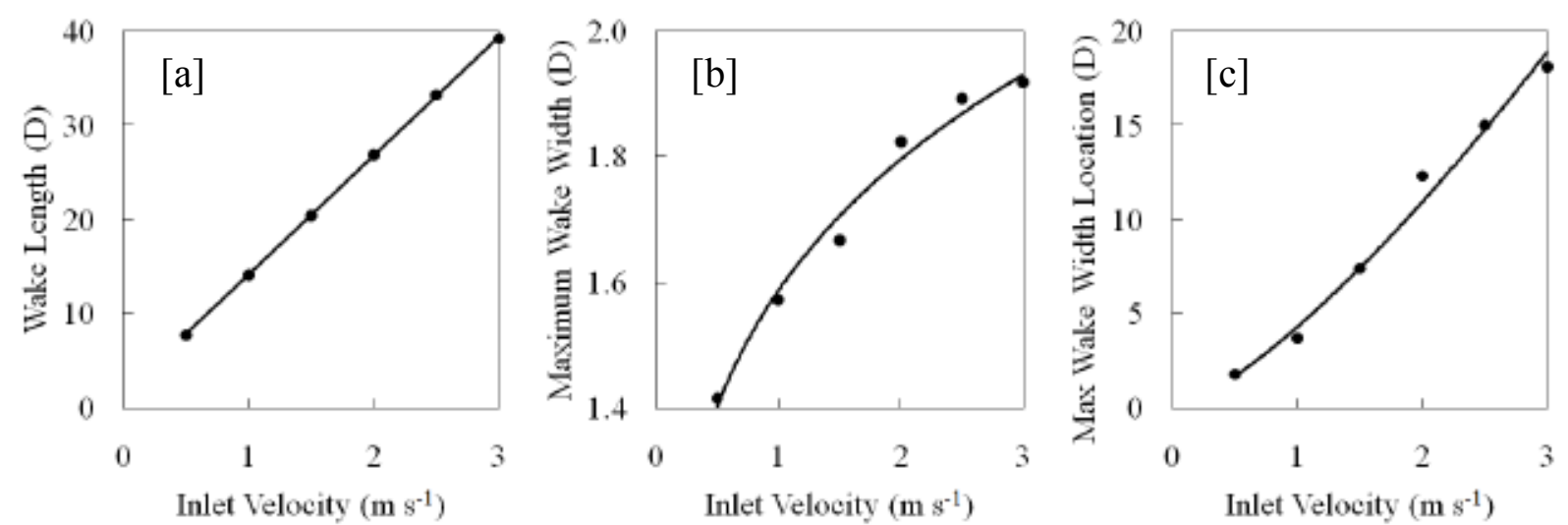

Figure 7: Variation in a) wake length; b) maximum wake width; and c) maximum wake width location with inlet velocity for a tip speed ratio of 3.0

\section{CONCLUSION}

A combined Blade Element Momentum - Computational Fluid Mechanics model was used for modelling a tidal stream turbine in a range of flow conditions typical of offshore aquatic environments. Simulation results were used to assess the influence of the turbine on flow structure and the size of the wake arising downstream.

The velocity downstream along the turbine centreline recovers to the free-stream velocity, and the distance required for flow recovery increases linearly with inlet velocity initially. However, above a certain free-stream velocity, the recovery distance length converges to a certain value. For instance, a recovery to a velocity deficit of 0.25 occurs over a distance equivalent to approximately 16.4 blade diameters for inlet velocities greater than $2.5 \mathrm{~m} \mathrm{~s}^{-1}$.

The influence of the turbines on the flow extends across the flow direction away from the centreline through the middle of the blade, and this was reflected in lateral velocity profiles. Immediately downstream of the blade, velocity deficits were slightly higher for lower velocities. However, for lower flow velocities, there was also a rapid reduction in velocity deficits with downstream distance.

These trends were evaluated by characterising the wake edge as having a velocity equivalent to $95 \%$ of the inlet velocity. Using this definition, variation in wake length, maximum wake width and wake expansion were evaluated. For the range of velocities considered, wake length increased linearly with velocity, and wake expansion continued over a longer distance downstream of the blade. The maximum wake width increased with velocity up to $3.0 \mathrm{~m} \mathrm{~s}^{-1}$, however, the wake width appeared to converge towards a constant value of approximately 1.92 diameters. Further work is required to assess the significance of transient flow features on the overall wake structure. Minor mesh-dependence was also identified and needs to be addressed.

It is widely believed that blade diameter is the most significant feature of a tidal turbine to influence the surrounding and downstream flow structure. The observations identified in this paper highlight that some wake characteristics are linearly related to the flow velocity, while others are not, or even converge towards a constant value. The key point is that the flow velocity is a significant parameter in determining wake characteristics. The relationships 
identified here can serve as a predictor of likely levels of wake interference between devices for different tidal turbine array arrangements to aid in layout planning.

\section{ACKNOWLEDGEMENT}

This work was undertaken as part of the Low Carbon Research Institute Marine Consortium (www.lcrimarine.org).

The Authors wish to acknowledge the financial support of the Welsh Assembly Government, the Higher Education Funding Council for Wales, the Welsh European Funding Office, and the European Regional Development Fund Convergence Programme.

\section{REFERENCES}

[1] P. Gilson, "Alstom's first Tidal In Stream Energy Converter Demonstration Project", in Proc. ICOE (2010).

[2] P.L. Fraenkel, "Marine current turbines: Pioneering the development of kinetic energy converters", in Proc. IMechE (Journal of Power and Energy), Vol. 221(2), pp. 159-169, (2007).

[3] M. Paish, J. Giles and B. Panahandeh, "The Pulse Stream Concept, and the development of the Pulse Stream Demonstrator", in Proc. ICOE, (2010).

[4] T. O'Doherty, D.A. Egarr, A. Mason-Jones and D.M. O'Doherty, "An assessment of axial loading on a five-turbine array", in Proc. ICE (Energy), 162 (EN2), pp. 57-65 (2009).

[5] I. Masters, J.C. Chapman, M.R. Willis and J.A.C. Orme, "A robust blade element momentum theory model for tidal stream turbines including tip and hub loss corrections", Journal of Marine Engineering and Technology, 10(1), pp. 25-35 (2011).

[6] B.E. Launder and D.B. Spalding, Mathematical models of turbulence, Academic Press, London (1972).

[7] H.K. Versteeg and W. Malalasekera, An Introduction to Computational Fluid Dynamics: The Finite Volume Method, Pearson Prentice Hall, London (2007).

[8] T.N. Croft, K. Pericleous and M. Cross, "PHYSICA: A multiphysics environment for complex flow processes", Numerical Methods in Laminar and Turbulent Flows, 9, Taylor C. et al. (Eds). Pineridge Press, Swansea (1995).

[9] S.V. Patankar, Numerical Heat Transfer and Fluid Flow, McGraw-Hill, New York, (1980).

[10] C.M. Rhie and W.L. Chow. "Numerical study of the turbulent flow past an aerofoil with trailing edge separation", American Institute of Aeronautics and Astronautics Journal, 22, pp 1525-1532 (1983).

[11] P. Chow, M. Cross and K. Periclious, "A natural extension of standard control volume CFD procedures to polygonal unstructured meshes", Applied Mathematical Modelling, 20, pp 170-183 (1995)

[12] L. Myers and A. Bahaj, "Design of 1st-generation marine current energy converter arrays", in Proc. WREC, (2010)

[13] Phase II UK Tidal Stream Energy Resource Assessment, Black \& Veatch, UK (2005). 